\title{
Toxoplasmosis: infección oportunista en pacientes con el síndrome de inmunodeficiencia adquirida.
}

\author{
Revisión
}

Ivonne Martín-Hernández ${ }^{1}$, Susana M. García-Izquierdo² .

${ }^{1}$ Laboratorio de Errores Innatos del Metabolismo. Centro Nacional de Genética Médica. ${ }^{2}$ TecnoSUMA Internacional. Cuidad de la Habana, Cuba.

\section{RESUMEN.}

Objetivos: Brindar al lector aspectos generales de la toxoplasmosis en pacientes con SIDA: patogenia, manifestaciones clínicas, diagnóstico, tratamiento y profilaxis.

Fuentes de extracción: Artículos actualizados y libros de texto relacionados con el tema. Consulta en Internet.

Resultados: La toxoplasmosis es la enfermedad causada por la infección con el parásito intracelular obligado Toxoplasma gondii. Constituye una infección oportunista en pacientes infectados con el VIH con menos de 100 linfocitos T CD4/ $\mu \mathrm{L}$. Esta parasitosis involucra una variedad de órganos del hospedero, particularmente el tracto gastrointestinal, pulmones, miocardio, cerebro y ojos. Entre el 10-50\% de los pacientes infectados con el VIH que son seropositivos para T. gondii desarrollan encefalitis toxoplásmica. La retinitis toxoplásmica es la segunda localización de la toxoplasmosis en pacientes con SIDA. Ante la sospecha clínica de neurotoxoplasmosis se recomiendan tomografía axial de cráneo o resonancia magnética. El diagnóstico definitivo es obtenido por biopsia y demostración del parásito. Dada la morbilidad y mortalidad de la toxoplasmosis en la población infectada con VIH, existe una gran necesidad de desarrollar tratamientos efectivos y profilaxis que permitan prolongar y mejorar la vida de estos pacientes.

Conclusiones: La frecuencia de esta parasitosis y los daños que ocasiona en los individuos inmunocomprometidos conllevan a la profundización en los conocimientos que se tienen a su respecto.

(Rev Biomed 2003; 14:101-111)

Palabras clave: Toxoplasmosis, SIDA, virus de inmunodeficiencia humana, encefalitis toxoplásmica, toxoplasmosis ocular.

Solicitud de sobretiros: Lic. Ivonne Martín Hernández, Centro Nacional de Genética Médica, Calle 146 No. 3102 esq. Ave 31 . Marianao C.P. 11600. Ciudad de la Habana. Cuba. Email: ivonne.martin@infomed.sld.cu

Recibido el 27/Febrero./2003. Aceptado para publicación el 7/Abril/2003. 


\section{Martín-Hernández, SM García-Izquierdo.}

\section{SUMMARY.}

Toxoplasmosis: oportunistic infection in patients with acquired immunodeficiency syndrome.

Objectives: To provide the reader with general aspects of toxoplasmosis in AIDS.

Sources of information: Updated articles and books related to the theme. Internet consultation.

Results: Toxoplasmosis is the disease caused by obligate intracellular parasite Toxoplasma gondii and constitutes an opportunistic infection in HIV-infected individuals with less than $100 \mathrm{CD} 4 \mathrm{~T}$ lymphocytes/ $\mu \mathrm{L}$. This parasitical infection involves a variety of host organs, particularly gastrointestinal tract, lungs, myocardium, brain and eyes. Between $10-50 \%$ of HIV-infected patients who are seropositive for $T$. gondii develop toxoplasma encephalitis. Toxoplasma retinitis is the second localization of toxoplasmosis in AIDS-patients. The demonstration of toxoplasmosis indicative serology is rarely possible. In the presence of neurotoxoplasmosis clinical suspicion computed tomography and magnetic resonance imaging are recomended. The definitive diagnosis is obtained by biopsy and parasite demonstration. Given the morbidity and mortality of toxoplasmosis in HIVinfected population, there is a great need to develop effective treatments and prophylaxis that permit the prolongation and improvement of these patients' lives. Conclusions: The frequency of this parasitical infection and the damage it causes in immunocompromised individuals implies the need for a profound analysis of it.

(Rev Biomed 2003; 14:101-111)

Keywords: Toxoplasmosis, AIDS, human immunodeficiency virus, toxoplasma encefalitis, ocular toxoplasmosis.

\section{INTRODUCCION.}

La Toxoplasmosis es la enfermedad causada por el parásito intracelular Toxoplasma gondii. Posiblemente la característica más importante, entre las biológicas, es su distribución universal, en todos los climas y en todos los grupos animales de sangre caliente (mamíferos y aves) $(1,2)$.
El virus de la inmunodeficiencia humana (VIH) provoca una disminución continua de linfocitos CD4+, causando un deterioro lentamente progresivo del sistema inmunológico en las personas infectadas. Una de las consecuencias de este hecho es la aparición de infecciones oportunistas con una importante morbilidad y mortalidad. La toxoplasmosis constituye generalmente una complicación tardía en los pacientes infectados con este virus y usualmente ocurre en pacientes con menos de 100 células T CD4/ $\mu \mathrm{L}(3,4)$. La infección primaria con $T$. gondii en hospederos inmunodeprimidos resulta en una infección letal diseminada, involucrando diversos órganos y tejidos como tracto gastrointestinal, pulmones, miocardio, cerebro y ojos. Esta infección oportunista aparece aproximadamente en el $15 \%$ de estos pacientes y es más común en individuos que viven en el Caribe y en Francia (5-8).

En pacientes con SIDA se puede producir una reactivación de la infección latente con $T$. gondii, cuya frecuencia depende de su prevalencia en la población general. Entre el 10-50\% de los pacientes infectados con el VIH y con serología positiva para Toxoplasma desarrollan encefalitis toxoplásmica (ET), con casi un $50 \%$ de secuelas neurológicas y cuya mortalidad se acerca al $20 \%$. Estos porcentajes la convierten en la segunda infección oportunista más común que afecta el sistema nervioso central (SNC) en pacientes VIH positivos. La mayoría de los casos de toxoplasmosis en enfermos con SIDA que se han reportado se relacionan con infección del sistema nervioso central, afectando el estado mental, la función neurológica, la visión, la audición y llegando hasta la muerte (9).

La toxoplasmosis es una infección rara en los niños infectados por VIH y la forma clínica más frecuente suele ser la toxoplasmosis congénita. El riesgo de toxoplasmosis congénita en niños nacidos de madres doblemente infectadas por T. gondii y VIH no está bien establecido; sin embargo, no parece existir una diferencia significativa entre los hijos de la mujer infectada por VIH y las no infectadas. La reactivación de la infección por T. gondii en la madre durante el embarazo representa un factor de riesgo importante para la transmisión intrauterina $(10,11)$.

\section{Revista Biomédica}




\section{Toxoplasmosis en pacientes con SIDA.}

\section{PATOGENIA.}

Los parásitos son liberados de los quistes intracelulares (bradizoitos) o de los oocistos (esporozoitos) por el proceso digestivo en el tracto gastrointestinal del hospedero. Se multiplican en los enterocitos y a continuación los trofozoítos formados se diseminan por el torrente sanguíneo o linfático y parasitan las células de una variedad de órganos, particularmente tejidos linfáticos, músculo esquelético, miocardio, retina, placenta y más frecuentemente el SNC. Penetran en las células de forma activa gracias a sus movimientos y a la producción de lisozimas y hialuronidasas; en algunas ocasiones lo hacen por un procedimiento similar a la fagocitosis. En estas células se multiplican por endodiogénesis, forman cúmulos citoplasmáticos y provocan lesiones tisulares como consecuencia de la destrucción celular y una reacción inflamatoria subsiguiente, que consiste típicamente en células mononucleares, algunos polimorfonucleares y edema. Este período de proliferación corresponde a la fase aguda de la toxoplasmosis. En el hospedero inmunodeprimido la respuesta inmune humoral y celular necesaria para controlar la expansión de los taquizoitos está afectada. Este estado inmune alterado conlleva a una progresiva destrucción focal que resulta en el fallo del órgano (ejemplo: encefalitis necronizante, neumonía y miocarditis) $(12,13)$.

En el hospedero inmunocompetente incluso después de la respuesta inmunológica efectiva, no se erradican los microorganismos; se forman algunos quistes en estos órganos desde la primera semana de la infección y permanecen latentes toda la vida del huésped. En los pacientes VIH positivos cuando se produce depresión de su sistema inmune, la proliferación activa de los microorganismos puede causar la reactivación de la enfermedad local y su diseminación. La localización de los quistes se encuentra con preferencia en las células del sistema nervioso central, coriorretina y músculos (esquelético y miocardio). Ocasionalmente los quistes pueden romperse y dejar en libertad los bradizoitos; si son muchos los que se rompen se produce una reactivación de la enfermedad, que puede ser localizada o generalizada $(12,13)$.
La inmunopatogénesis de la ET ha podido definirse con mayor precisión en los últimos años, llegando a establecerse algunos aspectos interesantes en torno al origen de la enfermedad. El sistema nervioso central está protegido contra los anticuerpos por la barrera hematoencefálica, carece de un sistema linfático y presenta niveles muy bajos de expresión de moléculas del complejo mayor de histocompatibilidad $(\mathrm{CMH})$. Los estudios experimentales han demostrado que el desarrollo de ET es un fenómeno regulado por los genes de la clase I del CMH. La vía de presentación de antígenos mediada por los linfocitos CD8+ está regulada por estas moléculas y, de esta forma, parece controlarse el número de quistes de $T$. gondii que sobrevivirán. El papel de los linfocitos es evidenciado por el hecho de que la ET se presenta generalmente cuando el recuento de células CD4+ ha caído por debajo de $100 / \mu \mathrm{L}$. Estos linfocitos, en conjunto con los CD8+ son capaces de liberar interleucinas (IL-2, Il-4, Il-5, IL-10 e interferón gamma) claves en la protección contra el patógeno. Otras interleucinas producidas por los macrófagos como IL-12 y la activación de las células asesinas naturales complementan el armamento para la defensa del organismo normal (12-16).

En el sistema nervioso central en particular, la respuesta sistémica es mínima. Sin embargo, los linfocitos T y B pueden ingresar allí para activar las células gliales, protagonistas de la defensa inmune en el cerebro. Los astrocitos, por ejemplo, son las células encargadas de la presentación de los antígenos del Toxoplasma, y la microglia ejerce la función fagocítica más importante. Las citocinas liberadas por los linfocitos que han penetrado al SNC además de activar la microglia, pueden inducir la producción de células citotóxicas que intentarán disminuir la replicación del parásito. En los pacientes con SIDA, los mecanismos celulares de defensa son insuficientes para controlar el crecimiento de $T$. gondii; de igual forma, la liberación de sustancias quimiotácticas y citocinas es insuficiente, de tal suerte que la invasión parasitaria persiste, produciendo además, una reacción inflamatoria local, responsable de muchos de los signos y síntomas de la enfermedad (12-16).

Vol.14/No. 2/Abril-Junio, 2003 


\section{Martín-Hernández, SM García-Izquierdo.}

La transmisión placentaria se realiza directamente a través de los vasos sanguíneos, con inflamación previa del corion, o provocando una placentitis con multiplicación en las células sincitiales. Posteriormente pasan a la sangre fetal por un mecanismo de pinocitosis. También se admite el paso a través del líquido amniótico por deglución fetal (11, 12).

\section{MANIFESTACIONES CLÍNICAS.}

La ET es la lesión más común que se presenta como masa en el SNC del paciente con SIDA y debe pensarse en neurotoxoplasmosis una vez que el infectado por el VIH presenta fiebre y signos neurológicos focales. La forma difusa de encefalitis toxoplásmica es rara en el SIDA, pero debe sospecharse cuando las otras causas de encefalitis fueron descartadas, estando indicado iniciar un tratamiento empírico de prueba. La mayoría de las veces se trata de una reactivación de la infección crónica latente que se disemina por vía hematógena y puede ocasionar lesiones multicéntricas con compromiso de los plexos coroides. Se ha descrito que el riesgo de padecer toxoplasmosis cerebral comienza cuando los linfocitos CD4 descienden por debajo de 200 células/ $\mu \mathrm{L}$, haciéndose máximo por debajo de 100 células/ $\mu \mathrm{L}$.

Los síntomas en los pacientes inmunodeprimidos con toxoplasmosis cerebral pueden ser focales o generalizados, siendo la cefalea, la confusión, la fiebre y convulsiones los más importantes. Clínicamente predomina un síndrome compatible con lesiones ocupantes; son comunes además hemiparesias, deficiencias visuales, hipertensión endocraneana, elementos neurológicos focales, compromiso de pares craneanos, trastornos de conciencia, alteraciones siquiátricas y somnolencia. Raramente se encuentra rigidez de nuca. La sintomatología es variable dependiendo de la localización de las lesiones y su número (19-21).

La toxoplasmosis ocular ocurre por la activación de los quistes depositados en la retina o cerca de ella. La retinitis focal necrotizante es la lesión característica. La retinitis toxoplásmica es la segunda localización de la toxoplasmosis en SIDA y es la segunda causa de retinitis en esta enfermedad. Posee una frecuente asociación con lesiones cerebrales (en $30 \%$ de los casos), hecho jamás observable en pacientes inmunocompetentes. La retinitis toxoplásmica en los pacientes con SIDA se presenta como una retinitis necrotizante, con uno o varios focos blanquecinos o blanco-amarillentos, de límites borrosos. La retina se halla engrosada, opaca y sus bordes no son granulares como los típicos de la retinitis por Citomegalovirus (CMV), sino homogéneos. En estos pacientes las lesiones son, en general, bilaterales y multifocales. Son excepcionales las escaras pigmentadas en uno de los bordes o lesiones satélites inactivas que típicamente se encuentran en pacientes inmunocompetentes, aunque es posible encontrarlas en un $4 \%$ de los casos. Crecen lentamente extendiéndose «en sábana» por la retina sin progresar hacia el vítreo, lo que las diferencia de las retinitis candidiásicas (ausencia de siembras vítreas), y sin «aclarar» y cicatrizar centralmente, como ocurre en las retinitis por CMV. Es casi constante la asociación de vitritis (70-100\%), y no es infrecuente que llegue a asociarse una uveítis anterior aguda granulomatosa (35-60\%) con ojo rojo y doloroso. Puede pasar inadvertida, siendo observable sólo si es expresamente buscada por bimicroscopía. La mayoría se localiza en el polo posterior (60\%) en la proximidad de los grandes vasos, pudiendo acompañarse de vasculitis. Pero cada vez se observan más casos de localización periférica lo que complica mucho el diagnóstico diferencial con las necrosis retinianas herpéticas incipientes. Pueden también asociarse ocasionalmente hemorragias, pero siempre son escasas y marginales. No es infrecuente observar un cierto desprendimiento de retina exudativo perilesional. Existen formas pseudotumorales con protusión de la lesión hacia el vítreo. Aunque excepcionalmente, pueden llegar a producirse tracciones vitreorretinianas y desprendimientos de retina regmatógenos (menos del $2 \%$ de casos), siendo la cuarta causa de desprendimiento de retina en pacientes con SIDA, después de la retinitis CMV, necrosis herpéticas y candidiasis (22-28).

Poco se ha publicado sobre la localización gastrointestinal de esta parasitosis. En pacientes con

\section{Revista Biomédica}




\section{Toxoplasmosis en pacientes con SIDA.}

toxoplasmosis gástrica los síntomas más frecuentes son pérdida de peso, anorexia, náuseas, vómitos, dolor abdominal en epigastrio, fiebre irregular, diarreas y síntomas digestivos altos. Suele aparecer en pacientes cuyos linfocitos T CD4 son menores que $50 / \mu \mathrm{L}(29$ $34)$.

\section{DIAGNÓSTICO.}

Ante la sospecha clínica de neurotoxoplasmosis se debe solicitar una tomografía axial computarizada (TAC) de cráneo. El hallazgo imagenológico de una o varias lesiones redondeadas, hipodensas con refuerzo anular post-contraste y edema perilesional reafirma la presunción diagnóstica. Aunque otras enfermedades pueden dar una imagen similar, son menos frecuentes. Los abscesos toxoplásmicos suelen localizarse en la unión córtico-subcortical y gangliobasal, pero pueden verse en otras topografías. Para la confirmación diagnóstica se debe identificar el germen mediante la realización de una biopsia aunque es riesgosa. En la práctica se admite que frente a la sospecha clínicoimagenológica, se inicie el tratamiento específico. La mejoría clínico-radiológica del paciente, en un plazo medio de 10 días, es un elemento a favor de ese diagnóstico etiológico. De no obtenerse mejoría, se sospecha que la causa de la lesión es otra y está indicado realizar una biopsia por punción aspirativa o a cielo abierto, enviando muestras para estudio al anatomopatólogo y al microbiólogo (35-40).

La resonancia magnética imagenológica (RMI) es más sensible para detectar pequeñas lesiones que no se visualizan en la TAC (38-40).

Raramente el diagnóstico se hace por detección del parásito en sangre, líquidos orgánicos o tejidos. Sin embargo, es posible detectarlo por técnicas histológicas y aislarlo en cultivos celulares o por inoculación al ratón. Por la técnica de reacción en cadena de la polimerasa (RCP) puede detectarse el ADN de T. gondii en tejidos y fluidos corporales. Cuando esta técnica se aplica a los tejidos (donde puede haber quistes) resulta imposible distinguir infección latente de activa, pero es válida para el estudio de sangre, líquido amniótico o líquido cefalorraquídeo, donde no hay quistes $(41,42)$.
Los niveles de anticuerpos anti-toxoplasma no tienen valor para el diagnóstico de esta parasitosis en el infectado por el VIH, aunque resulta útil el hallazgo de una serología negativa, pues aleja ese diagnóstico. La toxoplasmosis en estos pacientes puede ocurrir con bajos niveles de anticuerpos anti-Toxoplasma y raramente están presentes las IgM específicas. Esto se debe a la incapacidad del enfermo inmunodeprimido de formar nuevos anticuerpos durante las enfermedades agudas. El inmunodiagnóstico incluye ensayos enzimáticos sobre fase sólida (ELISA), aglutinación indirecta y Dye Test de Sabin-Feldman $(12,43)$.

El diagnóstico de coriorretinitis lo hace el oftalmólogo observando el fondo de ojo. Se confirma por la cicatrización obtenida después del tratamiento específico. Los exámenes complementarios no son indispensables para su diagnóstico. Si hay dudas puede ser útil realizar una angiografía retiniana con fluorescencia. La coriorretinitis por citomegalovirus es el diagnóstico diferencial más importante, especialmente en pacientes con SIDA.

El índice de Goldmann-Witmer-Desmonts, niveles de anticuerpos en vítreo o humor acuso respecto a los niveles de anticuerpos en sangre, puede ayudar al diagnóstico pero el porcentaje de positividades es inferior al de los pacientes inmunocompetentes. Su valor diagnóstico se incrementa notablemente cuando se combina con técnicas de RCP. La técnica de RCP en humor acuoso y/o vítreo, detecta regiones del ADN del T. gondii. Su especificidad en manos expertas es cercana al $100 \%$. Aunque la posibilidad de falsos positivos es mínima, persiste la posibilidad de falsos negativos al poder existir formas libres que no hayan quedado incluidas en la toma de muestra. Esta técnica en enfermos con SIDA puede llegar a presentar incluso mayor sensibilidad que en las retinitis de los sujetos inmunocompetentes dada la mayor incidencia de formas libres del parásito en los líquidos intraoculares. En casos excepcionales el diagnóstico será histológico, procedente de secciones de retina obtenidas por biopsia o tras enucleación, sobre las que también pueden aplicarse las técnicas de RCP (44-46). 


\section{Martín-Hernández, SM García-Izquierdo.}

\section{TRATAMIENTO.}

Debido a que la toxoplasmosis es reconocida como la mayor causa de morbidez neurológica y mortalidad en pacientes con SIDA constituye motivo de preocupación y controversia, haciéndose necesario un diagnóstico cuidadoso de la infección en el laboratorio, antes de indicar cualquier tratamiento.

No existe ningún tratamiento totalmente satisfactorio para combatir la toxoplasmosis. La toxoplasmosis puede ser tratada con variedad de drogas; la quimioterapia es supresiva de la proliferación toxoplásmica, pero no erradica la infección, es decir, no destruye los parásitos que se encuentran dentro de los quistes. Las drogas están por tanto dirigidas a las lesiones activas y ocasionalmente a disminuir la reacción inflamatoria. Debido a lo grave que es esta parasitosis, usualmente son necesarias por lo menos dos drogas (47).

Aunque se han descrito casos de curación espontánea de toxoplasmosis en individuos inmunocompetentes, en enfermos con SIDA la aparición de toxoplasmosis ocular es indicación absoluta de tratamiento, debiendo seguirse las mismas pautas terapéuticas que en la toxoplasmosis cerebral $(47,48)$.

Existen varios fármacos que han probado ser eficaces y entre ellos los más importantes son la pirimetamina, sulfonamidas y espiramicina. Otros como: azitromicina, clindamicina, claritromicina, trimetrexato, doxiciclina y atovacuona, pueden ser útiles en el tratamiento de la toxoplasmosis cerebral $(47,49)$.

La actividad de la espiramicina queda limitada a los taquizoitos, pues no atraviesa la membrana del quiste y, por lo tanto, no actúan sobre los bradizoitos. No obstante, algunos autores señalan que la espiramicina sí puede penetrar al interior del quiste. El tratamiento de selección es la asociación de pirimetamina y sulfonamidas. La espiramicina es menos tóxica, aunque menos activa que la pirimetamina. Puede emplearse asociada a ésta o a las sulfamidas y es el fármaco de elección para el tratamiento de la toxoplasmosis en el embarazo (47-50).

La pirimetamina se administra por vía oral, penetra bien en el líquido cefalorraquídeo (LCR) y produce bloqueo secuencial en el metabolismo del ácido fólico o folínico por activación de inhibidores enzimáticos en diferentes puntos de la vía metabólica. Es depresor de la médula ósea y puede ocasionar trombocitopenia y a veces anemia y leucopenia, por lo que en el transcurso del tratamiento deben realizarse controles de sangre periférica dos veces por semana. Para evitar su efecto tóxico, puede suministrarse ácido fólico por vía oral o intramuscular. Las personas con toxoplasmosis generalmente usan ácido folínico, una forma de ácido fólico para prevenir la anemia (47, 51).

Una combinación de pirimetamina y sulfadiacina, es eficaz para inhibir la replicación de trofozoítos y la posible diseminación durante los períodos en que se administran corticosteroides. El régimen de elección consiste en pirimetamina (100-200 mg de dosis inicial, después 50-100 mg/día) + ácido folínico 10 mg/día + sulfadiazina 2-8 g/día por vía oral mantenido durante al menos 6 semanas $(53,57,58)$. Aunque esta combinación puede causar una disminución de glóbulos blancos y problemas de riñón, es muy efectiva contra la toxoplasmosis. Más del $80 \%$ de las personas muestran mejorías después de 2 a 3 semanas de tratamiento $(47,51,52)$.

La más activa de las sulfamidas es la sulfadiacina, que se comporta de forma sinérgica con la pirimetamina, pudiendo además sustituirse por sulfonamidas triples (sulfameracina y sulfametacina) $(51,52)$.

La sulfadiacina es un medicamento tipo sulfa y aproximadamente la mitad de las personas que lo toman experimentan una reacción alérgica con síntomas como urticaria, picazón y náusea. Esta también se presenta como un salpullido y a veces causa fiebre. Las reacciones alérgicas pueden evitarse usando una técnica de desensibilización. Los pacientes comienzan a tomar dosis muy pequeñas del medicamento y luego van aumentando la cantidad hasta que llegan a tolerar la dosis completa. Las personas que no pueden tolerar medicamentos tipo sulfa pueden usar Clindamicina en lugar de sulfadiacina en la combinación a dosis de 900-1200 mg

\section{Revista Biomédica}




\section{Toxoplasmosis en pacientes con SIDA.}

administrados por vía intravenosa cada 6 horas ó 300$450 \mathrm{mg}$ por vía oral cada 6 horas, y manteniendo también el régimen durante al menos 6 semanas. Clindamicina puede causar diarrea a ciertas personas. En ambas combinaciones, los efectos secundarios son importantes y en muchos pacientes es imposible completar las 6-8 semanas de tratamiento $(47,51$, 52).

No se precisa un tratamiento específico para los pacientes con toxoplasmosis aguda sin ninguna otra anomalía, pero sí para los que presenten una sintomatología grave o una retinocoroiditis activa, en los cuales, la administración de un corticosteroide como la prednisona permite reducir el proceso inflamatorio y la cicatrización consiguiente de la retina. Fuera de esta indicación, los corticosteroides están contraindicados en el tratamiento de la toxoplasmosis. Los corticoides no están recomendados pues pueden favorecer la diseminación de los taquizoitos o reactivar focos latentes. Sin embargo, está justificado su empleo en los casos de retinocoroiditis, en los que los fenómenos de hipersensibilidad son importantes en el desarrollo de las lesiones (47).

En cuanto a la toxoplasmosis ocular, un régimen terapéutico eficaz es la combinación de clindamicina y sulfadiacina, pero hay que valorar los efectos colaterales potenciales de la clindamicina (como la colitis pseudomembranosa) y sopesarlos con los de la pirimetamina al considerar el posible uso de este régimen (47).

El cotrimoxazol (trimetropin + sulfametoxazol) ha probado ser eficaz pero con una actividad inferior a la asociación de la pirimetamina y sulfadiacina o pirimetamina y sulfonamidas triples. La terapia con cotrimoxazol no se recomienda con frecuencia debido a su intolerancia y efectos secundarios. Para este tipo de paciente se recomienda comenzar con un régimen de desensibilización $(47,53,54)$.

Otros posibles regímenes mantienen la pirimetamina y el ácido folínico, a los que añaden bien azitromicina, claritromicina o atovacuone. Como terapia de rescate se utiliza azitromicina a dosis de al menos 2 tomas orales de $900 \mathrm{mg}$ el primer día, luego $1200 \mathrm{mg} /$ día durante 6 semanas, y posteriormente 600 mg/día (los pacientes de menos de $50 \mathrm{~kg}$ de peso reciben la mitad de las dosis); por vía intravenosa se utilizan dos dosis de $500 \mathrm{mg}$ el primer día, luego $500 \mathrm{mg} /$ día durante 9 semanas, y finalmente el régimen oral (54).

Mientras se administran estas drogas debe evitarse el embarazo y la concepción siguiente debe ser por lo menos un mes después de finalizado el tratamiento. Hasta el momento, no se ha observado resistencia a las drogas por parte del parásito (56).

\section{PROFILAXIS.}

La experiencia que progresivamente se va adquiriendo en el manejo de pacientes con SIDA, la instauración de profilaxis, el tratamiento precoz de las infecciones oportunistas y los continuos avances en la terapia antirretroviral permiten prolongar y mejorar la vida de los pacientes con SIDA (55).

Dada la frecuencia de la toxoplasmosis en la población de infectados con el VIH, se ha hecho necesario el establecimiento de una profilaxis efectiva. En el caso de los pacientes VIH positivos en los que no se demuestre infección latente, las recomendaciones deben ser de carácter preventivo. Lo ideal sería disponer de un fármaco que fuera útil para prevenir esta infección y a la vez protegiera frente a otras infecciones oportunistas con el menor grado de toxicidad posible.

Para este tipo de pacientes seronegativos a Toxoplasma se les recomiendan las siguientes medidas higiénicas sanitarias:

Cocción adecuada de los alimentos y en especial de las carnes.

Beber agua potable.

Lavarse las manos con agua y jabón antes de ingerir alimentos.

Lavar las verduras y frutas antes de consumir.

Cuando trabaje con tierra protegerse con guantes y máscara.

Tener especial cuidado con los gatos. Preferiblemente este tipo de paciente debe evitar el contacto con los gatos y en especial con sus heces fecales. Si tiene que realizar el cambio de su arena higiénica, debe realizarlo con máscara y guantes. Después lavarse bien las manos. Mantener los gatos 


\section{Martín-Hernández, SM García-Izquierdo.}

dentro de la casa para que no salgan de cacería y alimentarlos con carnes bien cocidas (55-57).

Las personas con serología negativa a toxoplasma que no estén siendo tratadas con cotrimoxasol deberán volver a ser analizadas para el anticuerpo IgG contra toxoplasma cuando su recuento de linfocitos CD4 sea menor de 100 células/ $\mu \mathrm{L}$ para determinar si han seroconvertido y por tanto están en riesgo de contraer toxoplasmosis (55-57).

La finalidad de la quimioprofilaxis es prevenir la transformación de una enfermedad latente en enfermedad clínica (profilaxis primaria) o evitar la recidiva de la enfermedad (profilaxis secundaria). Aunque no es una pauta universalmente aceptada, en las zonas geográficas con alta incidencia de toxoplasmosis se está administrando profilácticamente tratamiento antitoxoplásmico en todos los VIH positivos con menos de $100 \mathrm{CD} 4 / \mu \mathrm{L}$. Las pautas y dosis son similares a las usadas en la quimioprofilaxis secundaria (56).

En estudios de profilaxis de la neumonía por PNC se ha visto que el cotrimoxazol es eficaz también en la profilaxis de la toxoplasmosis cerebral y las dosis diarias recomendadas son las mismas que en el caso de la PNC $(53,54)$.

Si los pacientes son intolerantes al tratamiento con cotrimoxazol, se recomienda como alternativa un régimen que también es eficaz contra la PNC: pirimetamina + dapsona. También puede considerarse el tratamiento profiláctico con atovaquona con o sin pirimetamina. Los datos de que se dispone actualmente no permiten recomendar monoterapia profiláctica con dapsona, pirimetamina, azitromicina o claritromicina $(56,58,59)$.

Las mujeres infectadas por el VIH con serología positiva frente a Toxoplasma transmiten más fácilmente el parásito al feto y la toxoplasmosis en ellos sigue un curso más rápido; por ello en las embarazadas cuyo recuento de linfocitos CD4+ esté por debajo de 200 células/ $\mu \mathrm{L}$ se aconseja la administración de $1 \mathrm{~g} / \mathrm{ocho}$ horas de espiramicina durante todo el embarazo (50). Después del tratamiento de la toxoplasmosis cerebral son frecuentes las recaídas ya que los fármacos empleados en el tratamiento son poco eficaces frente a los quistes. Por ello es necesario que los pacientes continúen el tratamiento inicial con un tratamiento de mantenimiento que evite las recidivas. Así, los pacientes con episodios previos de toxoplasmosis cerebral deben ser tratados con terapia supresiva de por vida. El tratamiento de elección para el mantenimiento se basa en la administración de pirimetamina, sulfadiazina y leucovorin. Un régimen alternativo utilizado en pacientes intolerantes a las sulfamidas es pirimetamina con clindamicina; sin embargo, solamente la combinación de pirimetamina con sulfadiazina proporciona también protección contra PNC $(59,60)$. En lo que respecta a la interrupción de la profilaxis secundaria, el número de pacientes que la han suspendido tras responder al tratamiento antirretroviral es insuficiente para poder recomendarla.

\section{REFERENCIAS.}

1.- Tenter AM, Heckeroth AR, Weiss LM. Toxoplasma gondii: from animals to humans. Int J Parasitol 2000; 30: 1217-58.

2.- Remington JS, McLeod R., Desmonts G. Toxoplasmosis. En: Remington JS, Klein JO, editores. Infectious diseases of the fetus and newborn. 5a ed. Philadelphia: W.B. Saunders; 2001.p. 205-346.

3.- Kitahata MM, Koepsell TD, Deyo RA. Physician's experience with adquired immunodeficiency syndrome as a factor in patient's survival. N Engl J Med 1996; 334: 701-6.

4.- Okome-Nkoumou M, Mbounja-Loclo ME, Kombila M. Spectrum of opportunistic infections in subjects infected with HIV. Santé 2000;10: 329-37.

5.- Wong S Y, Remington J. S.: Biology of Toxoplasma gondii, AIDS, 1993; 7: 299-318.

6.- Maïga I, Kiemtoré P, Tounkara A. Prévalence des anticorps antitoxoplasmiques chez les malades atteints du syndrome d'immunodéficience acquise et les donneurs de sang à Bamako. Bull Soc Pathol Exot 2001, 94 (3): 268-70.

7.- Góngora-Biachi RA, Gónzalez-Martínez P, CastroSansores C, Lara-Perera D, Alonzo G, Pavía N, et al. Prevalencia de anticuerpos contra Toxoplasma gondii en donadores de sangre y pacientes con la infección del virus de la inmunodeficiencia humana en Yucatán, México. Enf Infec Microbiol 1996; 16:13-6.

\section{Revista Biomédica}




\section{Toxoplasmosis en pacientes con SIDA.}

8.- Góngora-Biachi RA, Gónzalez-Martínez P, CastroSansores C, Alvarez R, Pavia-Ruz N, Lara-Perera D, et al. Anticuerpos contra Toxoplasma gondii en pacientes con VIH en Yucatán. Rev Invest Clin 1998; 50: 419-22.

9.- Luft BJ, Hafner R, Korzun AH. Toxoplasmic encefalitis in patients with adquired immunodeficiency syndrome. N Engl J Med 1993; 329: 995-1000.

10.- Couvreur J, Thulliez P, Daffos FD. Toxoplasmosis. Manual de enfermedades Infecciosas. Infecciones obstétricas y perinatales. 2a ed. México: Mosby Doyma Libros; 1994. p.160-81.

11.- Petersen E, Pollak A, Reiter-Owona I. Recent trends in research on congenital toxoplasmosis. Int J Parasitol 2001; $31: 115-44$

12.- Kasper LH. Toxoplasma infection. En: Braunwald E, Fauci A, Kasper DL, Hauser SL, Longo DL, Jameson JL, editors. Harrison's Principles of Internal Medicine. 15 ed. New York: McGraw-Hill; 2001.p. 1222-7.

13.- Fauci A, Lane HC. Human Immunodeficiency virus (HIV) disease: AIDS and related disorders. En: Braunwald E, Fauci A, Kasper DL, Hauser SL, Longo DL, Jameson JL, editors. Harrison's Principles of Internal Medicine. 15 ed. New York: McGraw-Hill; 2001.p. 1852-913.

14.- Chardes T, Buzoni D, Lepage A. Toxoplasma gondii oral infection induces specific cytotocic CD8 a Thy 1 gut intraepithelial lymphocytes lytic for parasites infected enterocytes. J Immunol 1994; 50: 4596-603.

15.- Chardes T, Bout D. Mucosal immune response in toxoplasmosis. Res Immunol 1993 ; 144 : 57-60.

16.- Purner M, Berens R, Tomavo S, Lecordier L, CesbronDelauw MF, Kotzin B, et al. Stimulation of human lymphocytes obtained from Toxoplasma gondii-seronegative persons by proteins derived from T. gondii. J Infec Dis 1998; 177: 746-53.

17.- Cochereau-Massin I, LeHoang P, Lautier-Frau M. Ocular toxoplasmosis in human immunodeficiency virus-infected patients. Am J Ophthalmol 1992; 114: 130-5.

18.- Hernández O, Braselli A, Purtscher H. Toxoplasmosis encefálica en pacientes con síndrome de inmunodeficiencia adquirida (SIDA). Rev Med U 1990; 6(3): 192-6.

19.- McCabe R, Chirurgi V. Issues in toxoplasmosis. Infect Dis N A 1993; 7: 587-604.
20.- Frenkel JK. Toxoplasmose. En: Veronesi R., Focaccia, editores. Tratado de Infectología. Sao Paulo: Atheneu; 1997. p.1290-305.

21.- Guerrero-Flores A. Encefalitis toxoplásmica en el SIDA. Treinta y nueve casos clínicos. Enferm Infec Microbiol 1997; 17: 65-7.

22.- Holland G, Engstrom RE, Glasgow BJ. Ocular toxoplasmosis in patients with the acquired immunodeficiency syndrome. Am J Ophthalmol 1988; 106: 653-67.

23.- Autelitano A. Diffuse necrotizing retinochoroiditis in a child with AIDS and toxoplasmic encephalitis. Graefe's Clin Exp Ophthalmol 1990; 228: 36-9.

24.- Grossniklaus HE, Specht CS, Allaire G. Toxoplasma gondii retinochoroiditis and optic neuritis in acquired immune deficiency syndrome: report a case. Ophthalmol 1990; 97: $1342-6$

25.- Berger BB, Egwuagu CE, Freeman WR. Military toxoplasmic retinitis in acquired immunodeficiency syndrome. Arch Ophthalmol 1993;111: 373-6.

26.- Moorthy RS, Smitth RE, Rao NA. Progressive ocular toxoplasmosis in patients with acquired immunodeficiency syndrome. Am J Ophthalmol 1993; 115:742-7.

27.- Pivetti-Pezzi P, Accorinti M, Tamburi S. Clinical features of toxoplasmic retinochoroiditis in patients with acquired immunodeficiency syndrome. Ann Ophthalmol 1994; 26: 73 84.

28.- Oréfice F, Bonfioli AA. . Uveíte clínica e cirúrgica. En: Oréfice F, editor. Toxoplasmose. Rio de Janeiro: Editora Cultura Médica; 2000. p. 619-80.

29.- Smart P, Weinfel d A, Tompson NE. Toxoplasmosis of the stomach : a cause of antral narrowing. Radiology 1990; 174:369-70.

30.- Florencio RFC, Alburquerque Filho FB, Moraes MAP. Toxoplasma gondii na mucosa gástrica como primeiro achado em paciente aidetico. Rev Soc Brasil Med Trop 1992;25: 275-6.

31.- Peraire J, Vidal F, Mayayo E. Gastric toxoplasmosis in the acquired immunodeficiency syndrome. Am J Gastroenterol 1993; 88: 1464-5.

32.- Alpert L, Millar M, Alpert E. Gastric toxoplasmosis in 


\section{Martín-Hernández, SM García-Izquierdo.}

the acquired immunodeficiency syndrome: antemortem diagnosis with histophatologic characterization. Am J Gastroenterol 1996; 110: 258-64.

33.- Kofman E, Khorsandi A, Sarlin J. Gastric toxoplasmosis: case report and review of the literature. Am J Gastroenterol 1996; 91: 2436-38.

34.- Guerrero-Flores A, Vega-Ramos B. Toxoplasmosis gástrica en el síndrome de inmunodeficiencia adquirida. Rev Biomed 2002; 13:37-41.

35.- Mirlesse V, Jacquemard F, Daffos F. Toxoplasmose au cours de la grossesse. Diagnostic et nouvelles posibilités thérapeutiques. Presse Méd. 1993; 22:258-62.

36.- Ammasari A, Murri R, Cingolani A, De Luca A, Antinori A. AIDS-Associated Cerebral Toxoplasmosis En: Gross U, editor. Toxoplasma gondii: An Update on diagnosis and treatment. Berlin : Springer; 1996. p. 209-222.

37.- Renold C, Sugar A, Chave JP. Toxoplasma encephalitis in patients with the acquired immunodeficiency syndrome. Medicine 1992; 71: 224-39.

38.- Ciricillo SF, Rosenblum ML.Use of CT and MR imaging to distinguish intracranial lesions and to define the need for biopsy in AIDS patients. J Neurosurg 1990; 73:720-4.

39.- Katlama C. Diagnosis and treatment of toxoplasmosis of the CNS in patients with AIDS. CNS Drugs 1996; 5: 331-343.

40.- Raffi F, Aboulker JP, Michelet C, Reliquet V, Pelloux H, Huart A, et al. A prospective study of criteria for the diagnosis of toxoplasmic encephalitis in 186 AIDS patients. AIDS 1997; 11: 177-84.

41.- Montoya MT, Gómez JE, Castaño JC, Marx C, Aubert D, Bonhomme A, et al. Avances diagnósticos en Toxoplasmosis. PCR, nuevos marcadores de infección evolutiva y otras técnicas. Acta Med Colomb 1996; 21: 127-38.

42.- Brézin AP, Egwuagu CE, Burnier M. Identification of Toxoplasma gondii in paraffin-embedded sections by the polymerase chain reaction. Am J Ophthalmol 1990;110:599.

43.- Hayde M, Salzer HR, Gittler G, Aspock H, Pollak A. Microparticle enzyme immunoassay (MEIA) for Toxoplasma specific immunoglobulin $\mathrm{G}$ in comparison to the SabinFeldman dye test. A pilot study. Wien Klin Wochenschr 1995; 107:133-6.

44.- Vincent K, Van Doornik C, Ongkosuwito J, feron EJ, Kiljstra
A. Differences between intraocular and serum antibody response in patients with ocular toxoplasmosis. Am J Ophthalmol 1998; 126: 698-706.

45.- Chan CC, Palestine AG, Li Q. Diagnosis of ocular toxoplasmosis by the use of immunocitology and the polymerase chain reaction. Am J Ophthalmol 1994;117: 80345.

46.- Manners RM, O'Connell S, Guy EC. Use of polymerase chain reaction in the diagnosis of acquired ocular toxoplasmosis in an immunocompetent adult. Br J Ophthalmol 1994;78:583-4.

47.- Valdés MC, Díaz AG, Svarch N. Actualidades en el tratamiento y profilaxis de la toxoplasmosis. Rev Cubana Med Gen Inegr 1996;12: 4-6.

48.- Schimkat M, Althaus C, Armbrecht C. Treatment of ocular toxoplasmosis with atovaquone in a patient with AIDS. Klin Monastsbl Augenheilkd 1995;206: 173-7.

49.- Podzamczer D, Miro JM, Bolao F. Twice-weekly maintenance therapy with sulfadiazine-pyrimethamine to prevent recurrent toxoplasmic encephalitis in patients with AIDS. Spanish Toxoplasmosis Study Group. Ann Intern Med 1995; 123: 175-80.

50.- Guerina NG, Hsu H, Meissner HC, Maguire JH, Lynfield R, Stechenberg B. Neonatal screening and early treatment for congenital Toxoplasma gondii infection. N Engl J Med 1994; 330: 1858-963.

51.- Payen MC, Dewit S, Sommereijns B, Clumerk N. A controlled trial of dapsone versus pyrimethamine-sulfadoxine for primary prophylaxis of Pneumocystis carinii, pneumonia and toxoplasmosis in patients with AIDS. Biomed Pharm 1997; 51: 439-45.

52.- Podzamczer D, Miro JM, Ferrer E, Gatell JM. Thrice-weekly $v s$. daily sulfadiazine-pyrimethamine (SP) for maintenance therapy of toxoplasmic encephalitis (TE). 5th Conference on Retroviruses and Opportunistic Infections, Chicago, IL; January 31 to February 5, 1998. Abstract 468.

53.- Carr A, Tindall B, Brew BJ. Low-dose trimethoprimsulfamethoxazole prophylaxis for toxoplasmic encephalitis in patients with AIDS. Ann Intern Med 1992; 117: 106-11.

54.- Lane HC, Laughon BE, Falloon J, Kovacs JA, Davey RT, Polis MA. Recent advances in the management of AIDSrelated opportunistic infections. Ann Intern Med 1994; 120:945-55. 
55.- Gallart JE, Moore RD, Chaisson RE. Prophylaxis for opportunistic infections in patients with HIV infection. Ann Intern Med 1994; 120: 932-44.

56.- Iñigo MA, Sánchez A, Gónzalez M. Profilaxis de las infecciones oportunistas asociadas al SIDA. Farm Hosp 1996; 20:337-42.

57.- Bowie WR, King AS, Werker DH, Isaac-Renton JL, Bell A, Eng SB, et al. Outbreak of toxoplasmosis associated with municipal drinking water. Lancet 1997, 350: 173-7.

58.- Leport C, Chene G, Morlat P. Pyrimethamine for primary prophylaxis of toxoplasmic encephalitis in patients with human immunodeficiency virus infection: a double-blind, randomized trial. J Infect Dis 1996; 173: 91-7.

59.- Antinori A, Murri R, Ammassari A. Aerosolized pentamidine, cotrimoxazole and dapsone-pyrimethamine for primary prophylaxis of Pneumocystis carinii pneumonia and toxoplasmic encephalitis. AIDS 1995; 9: 1343-50.

60.- Podzamczer D, Salazar A, Jimenez J, Consiglio E, Santin $\mathrm{M}$, Casanova A, et al. Intermittent trimethoprimsulfamethaxozole compare with dapsone-pyrimethamine for the simultaneous primary prophylaxis of Pneumocystis pneumonia and toxoplasmosis in patients infected with HIV. Ann Intern Med 1995;122: 755-61. 